\title{
On Watson's Non-Forcing Contracts and Renegotiation*
}

\author{
Roberto Serrano ${ }^{\dagger}$ \\ Working Paper No. 2004-03
}

May 2004

\begin{abstract}
Watson (2002) proposes non-forcing contracts as a way to show the limitations of the mechanism design program with ex-post renegotiation (Maskin and Moore (1999)). If one takes a partial implementation approach, as Watson does, we show that non-forcing contracts do not constitute an intermediate paradigm between implementation with no renegotiation and with ex-post renegotiation. Moreover, taking a full implementation approach, non-forcing contracts fail if and only if one goes outside of the constraints identified by Maskin and Moore, because of the appearance of undesirable equilibria.
\end{abstract}

JEL classification numbers: C70, D74, K10.

Keywords: contracts, renegotiation, mechanism design.

${ }^{*}$ I owe special thanks to Eric Maskin. This paper could not have been written without many conversations with him on the subject. This research was supported by the National Science Foundation and Deutsche Bank. The author also thanks the Institute for Advanced Study for its hospitality.

${ }^{\dagger}$ Brown University, roberto_serrano@brown.edu, www.econ.brown.edu/faculty/serrano 


\section{Introduction}

Following the pioneering work of Hart and Moore (1988), the limitations that the possibility of renegotiation brings to the theory of implementation were first explored in Maskin and Moore (1999) [MM in the sequel]. Instead of allowing arbitrary mechanisms, MM argue that one should question the use of inefficient outcomes in the mechanism, because they could be renegotiated by the agents. This is especially compelling when one is considering mechanisms in the form of contracts between only two completely informed parties.

Watson (2002) [W from now on] challenges the approach taken by MM because, in his view, in many settings it does not give the right answer in terms of what is the set of implementable payoffs after renegotiation. His point is that the MM approach, by abstracting from the technological details of renegotiation, fails to adequately describe the set of implementable payoffs with renegotiation. In particular, he distinguishes between "public actions" and "inalienable actions" taken by parties, and argues that the standard approach is misguided in treating inalienable actions as if they were public. To make his point, he proposes non-forcing contracts as a way to expand the set of payoffs that are implementable with renegotiation. In his non-forcing contracts, the vehicle that makes the final outcomes be elements of the ex post Pareto frontier is not renegotiation, but the optimal actions of one agent as part of his inalienable decisions. Thus, W's logic implies that renegotiation is not necessary because optimal choices in a one-person decision problem take care of inefficiencies.

Given the extensive use of the MM methodology in contract theory, W's claim deserves to be carefully evaluated. ${ }^{1}$ We do so in this short paper, and conclude that the interpretation of renegotiation made in $\mathrm{W}$ is far too restrictive. There are plausible ways to model renegotiation under which the conclusions reached by MM are perfectly valid.

The main example in $\mathrm{W}$ features a non-durable trading opportunity (i.e., one that will expire at some future date). However, this should not affect renegotiation, which can start well before that expiration date, as soon as an ex post inefficiency is identified by the contracting parties.

Regardless of the durability of the trading opportunity, if one follows the partial implementation approach, we find that the non-forcing contracts proposed in $\mathrm{W}$ to support extra payoffs do not constitute an intermediate paradigm between "implementation without renegotiation" and that in MM: effectively, since renegotiation is never used in the analysis, the sets of partially implementable payoffs when using non-forcing contracts and when there is no renegotiation coincide.

Furthermore, non-forcing contracts give rise to multiple equilibrium outcomes. Since one possible path of play in the non-forcing contract is inefficient (following a non-optimal action on the part of an agent), the induced game is not one of constant sum. Therefore, one should be concerned with full implementation. From this point of view, W's non-forcing contracts fail if and only if the payoff to be implemented falls outside of the set identified by MM. That is, W's reductionist approach of replacing the renegotiation game with a one-person (inalienable) decision problem misses that the individual decision problem in question cannot be analyzed in isolation. Rather, the beliefs of that agent about how the other agent will behave matter and create the undesired multiplicity of equilibrium outcomes.

\footnotetext{
${ }^{1}$ Papers in the literature that adopt this approach include Che and Hausch (1999), Edlin and Reichelstein (1996), Segal (1999), and Segal and Whinston (2002).
} 
After his main example, W later extends his criticism by showing that in certain settings the set of implementable payoffs using only forcing contracts is a strict subset of the one that relies on both forcing and non-forcing contracts. We choose to concentrate on the example, although our arguments can also be extended to counter his more general results.

\section{Watson's Example}

There are two agents. Agent 1, the buyer, is considering whether she should buy an indivisible object from agent 2 , the seller. The two parties sign a contract that must specify whether or not trade should take place and a monetary transfer, as a function of verifiable information. In principle the contract (or mechanism) allows certain messages to be sent or actions to be taken, as the verifiable pieces of information.

After they sign the contract and before they send any verifiable messages, parties (either the buyer or the seller) may make an unverifiable investment that will determine the quality of the relationship. This quality will be observable to both parties, but unverifiable by an outside party. It is assumed that if there is investment, the quality of the good is high, and it is low otherwise. The cost of the investment is less than the relationship's total ex-post surplus in the high state after investment, which makes investment the efficient decision. However, Watson's focus is the characterization of implementable contracts after the investment decision has been made, and therefore, investment costs are sunk from this point of view.

Thus, there are two states of the relationship: "high" $(H)$ and "low" $(L)$, common knowledge among the two parties but unknown to outsiders. The design of a contract in such circumstances falls squarely under implementation with complete information.

In both states, the total surplus after investment generated by "no trade" is 0 . If the state is $L$, the total surplus after investment associated with "trade" is also 0 , while it is 8 in state $H, 5$ units of which go to the buyer and 3 to the seller.

The payoff to party $i(i=1,2)$ is simply the sum of the share in surplus that party $i$ receives and the monetary transfer (minus the cost of investment, in the case of the investing party).

\subsection{Efficient Implementable Payoffs}

The first question to address is what payoffs must the contract specify in order to induce ex-post efficient actions in both states: it will suffice to consider contracts that implement "trade" in state $H$ and "no-trade" in state $L$. To simplify, let us add the mild requirement that if the seller does not invest, both parties can walk away from this relationship with a zero payoff. This will ensure that there will be no monetary transfer in state $L$.

We begin with the partial implementation question, i.e., to find a contract where the desired payoffs in each state can be supported by an equilibrium. One can then appeal to the revelation principle and restrict attention to direct mechanisms. Thus, after the investment decision, both parties are asked to submit a simultaneous report of the state. If the report profile is $(h, h)$, trade is enforced and a monetary transfer of $p^{h h}$ from the buyer to the seller must take place. If the profile is $(l, l)$, "no-trade" and a zero transfer of money are enforced. If the reports are $(h, l)$ or $(l, h)$, there is no trade and monetary transfers $p^{h l}$ or $p^{l h}$ take place, respectively. 
We are interested in finding values for $p^{h h}, p^{h l}$ and $p^{l h}$ so that $(h, h)$ is a Nash equilibrium of the induced game following investment, and $(l, l)$ is a Nash equilibrium of the game following lack of investment.

Recall that we are referring to the buyer as agent 1 , while the seller is agent 2 . Then, one can write down the following conditions:

- For $(h, h)$ to be a Nash equilibrium of the game that follows investment, one must have:

$$
\begin{gathered}
5-p^{h h} \geq-p^{l h} \\
3+p^{h h} \geq p^{h l} .
\end{gathered}
$$

- For $(l, l)$ to be a Nash equilibrium of the game that follows lack of investment:

$$
\begin{gathered}
0 \geq-p^{h l} ; \\
0 \geq p^{l h} .
\end{gathered}
$$

All these constraints reduce to $p^{h h} \in[-3,5]$, thereby yielding ex-post payoff profiles (5 $\beta, 3+\beta)$ for any $\beta \in[-3,5]$.

It is not difficult to see that the same set of efficient payoffs is implementable if one uses the more demanding requirement of full implementation (given a specific payoff profile in each state, the contract's unique equilibrium payoff in each state coincides with it).

\subsection{Ex Post Renegotiation}

Following MM, one can argue that agents have an incentive to renegotiate those outcomes prescribed by the contract that are ex-post inefficient. Upon reading the contract, agents will be able to identify its inefficient final outcomes, and communication will take place between them that will lead to the elimination of such ex post inefficiencies. That is, as soon as an ex-post inefficient outcome is specified by the contract, the possibility of playing a bargaining game between both parties opens up. The MM view here, influenced by Coasian logic, is that bargaining under complete information will eventually yield an efficient outcome. Of course, the possibilities offered by this renegotiation game are numerous, and the final conclusions will depend on the specific game adopted. Let us suppose that the two parties' bargaining weights are exogenously specified, so that in the bargaining game the buyer will end up with $\pi_{1} \geq 0$ of the available total surplus after investment, while the seller will end up with $\pi_{2}=1-\pi_{1} \geq 0$. Following MM, it will be assumed that the renegotiation function (i.e., the value of $\pi_{1}$ in this case) is known to the contract designer.

In the direct contract specified in the previous subsection, there are three inefficient outcomes that the parties will want to renegotiate: these happen after investment, if the reported profile is $(l, l),(h, l)$ or $(l, h)$. The question is how this renegotiation will limit the set of implementable efficient payoffs. To address this, we appeal again to the revelation principle and assume that the agents play a direct mechanism.

The only difference now is that we have to be careful in specifying the outcomes after the profiles $(l, l),(h, l)$ or $(l, h)$ are reported, because, if inefficient, they will be renegotiated in state 
$H$. To make the set of efficient payoffs that are implementable after renegotiation with bargaining weights $\left(\pi_{1}, 1-\pi_{1}\right)$ as large as possible, it turns out that one must require two inequalities.

The first inequality gives us the upper bound for $p^{h h}$ and it comes from the buyer's incentive constraint:

$$
5-p^{h h} \geq \min \left\{5,8 \pi_{1}\right\}-p^{l h} .
$$

That is, following reports $(l, h)$, we enforce a monetary transfer of $p^{l h}$ from the buyer to the seller. In addition, we enforce trade if $\pi_{1} \geq 5 / 8$ and no renegotiation is necessary, while we prescribe "no-trade" if $\pi_{1}<5 / 8$, outcome that is renegotiated. Since from state $L$, we know that $p^{l h} \leq 0$, the above inequality yields

$$
p^{h h} \leq 5-\min \left\{5,8 \pi_{1}\right\} .
$$

The lower bound on $p^{h h}$ comes from the seller's incentive constraint:

$$
3+p^{h h} \geq \min \left\{3,8\left(1-\pi_{1}\right)\right\}+p^{h l} .
$$

That is, following reports $(h, l)$, apart from a transfer of $p^{h l}$, the contract must enforce "trade" if $\pi_{1}<5 / 8$, and prescribe "no-trade" otherwise. Using also the fact that $p^{h l} \geq 0$ as we know from state $L$, we obtain that

$$
p^{h h} \geq \min \left\{3,8\left(1-\pi_{1}\right)\right\}-3 .
$$

Therefore, we end up with the following characterization of efficient implementable payoffs after renegotiation when the bargaining weights are $\left(\pi_{1}, 1-\pi_{1}\right)$. To enhance comparison with the implementable payoffs before renegotiation, we write the typical profile of ex-post payoffs also as $(5-\beta, 1+\beta)$ :

- When $\pi_{1}<5 / 8$ : any $\beta \in\left[0,5-8 \pi_{1}\right]$.

- When $\pi_{1} \geq 5 / 8$ : any $\beta \in\left[5-8 \pi_{1}, 0\right]$.

For example, if $\pi_{1}=1 / 2$, as $\mathrm{W}$ assumes, the range of $\beta$ s compatible with efficient implementable payoffs after renegotiation is the interval $[0,1]$.

Note that ex-post renegotiation and the quasilinear structure of preferences turn the contract with renegotiation into a constant sum game. Therefore, the answer to the partial and full implementation questions continues to be the same.

\section{$2.3 \quad$ Non-Forcing Contracts}

W claims that the characterization of implementable payoffs after renegotiation performed by the MM approach is incorrect. He argues that in situations like in the example it does not give the right answer, in that one can actually get to implement a larger set of payoffs by making use of non-forcing trade options as contracts. To illustrate his claim, he assumes that the trading opportunity is non-durable, i.e., the trade decision, "trade" or "no-trade," cannot be reversed.

Suppose then that the trading opportunity is non-durable and consider the following contract. After the investment decision, the buyer decides to take delivery of the good or not. If not, "notrade" and no transfer are enforced; and otherwise, "trade" and a monetary transfer of $p$ from the buyer to the seller are enforced. 
W argues that the reason why the MM approach gives the wrong answer is that it restricts attention to "forcing contracts." Further, he asserts that, with renegotiation, one can expand the set of implementable payoffs if one uses "non-forcing" contracts. This would correspond to the trade option contract just defined where $p \in[0,5]$ : namely, if $p$ is in this interval, one will see the outcome "trade" in state $H$ and "no-trade" in state $L$ chosen by the buyer (whereas the buyer would be "forced" to trade or not to trade, regardless of the state, outside of this interval).

Therefore, although renegotiation is allowed, it is not necessary. It is up to the buyer to take care of the inefficiency by simply taking delivery in state $H$. Furthermore, it is in his incentive to do so as long as $p \leq 5$. Note the change in logic with respect to MM: the claim is that it is the optimal solution to this one-person decision problem (the buyer's in state $H$ ) that eliminates inefficiencies, leaving no room for renegotiation. By doing this, the non-forcing trade option contract has expanded the set of efficient partially implementable ex-post payoffs with renegotiation to be $(5-\beta, 3+\beta)$ for any $\beta \in[0,5]$, a strict superset of the interval $[0,1]$ that would arise in the MM model if bargaining weights are equal.

Upon comparing the different ranges of $\beta \mathrm{s}$, it would appear, and this is the way $\mathrm{W}$ asserts it, that the possibility of considering non-forcing contracts creates an intermediate paradigm between the implementation with no renegotiation of Subsection 2.1 and the MM approach of Subsection 2.2.

\section{Two Replies}

\subsection{Partial Implementation}

Taking W's partial implementation approach, we first address the question of whether non-forcing contracts uncover a new paradigm, intermediate between implementation without renegotiation and MM's.

To answer this, consider a different trade option contract, where after the investment decision, it is the seller who must choose whether trade should or should not take place.

Specifically, in this new trade option contract, the seller will be paid nothing if he chooses not to deliver the good, and will be paid a price $p$ otherwise. Insisting on making this contract non-forcing, as $\mathrm{W}$ does, implies that the possible prices allowed in it are those $p \in[-3,0]$. Again, only if $p$ lies outside of this interval, the seller will be "forced" to deliver (if $p>0$ ) or not to deliver (if $p<-3$ ) regardless of the state.

That is, the consideration of non-forcing contracts does not constitute an intermediate paradigm between no-renegotiation and the MM approaches. Appealing to these two different nonforcing contracts (trade options exercised by the buyer or by the seller) already yield the entire set of efficient implementable payoffs with no renegotiation, i.e., the payoffs $(5-\beta, 3+\beta)$ for any $\beta \in[-3,5]$.

The explanation for this is simple enough. Renegotiation is never used as part of the mechanism, and therefore, the set of partially implementable payoffs must be the one identified in Subsection 2.1. 


\subsection{Full Implementation}

Although $\mathrm{W}$ argues that he is not focusing on full implementation (see his footnote 11), one can no longer provide for this the argument that the game is one of constant sum, and that therefore, performing partial implementation is without loss of generality. In particular, in the trade option contract where the buyer makes the final decision (the contract discussed in our Subsection 2.3), the outcome "not take delivery" in state $H$ is feasible and inefficient. If renegotiation is precluded or ineffective, the presence of this outcome turns the game into one where the sum of payoffs is non-constant. One should then check the implications of full implementability in this contract.

Consider now the trade option where the buyer makes the decision. Let us look at a possible history in which the seller makes the investment. Suppose we interpret non-durability to mean that the trade opportunity will expire at some future time $t^{*}$, but that until that time, the trade decision can be reversed. In other words, it is possible for the two parties to play a renegotiation game in the interval of time prior to $t^{*}$. Again, in general it will matter how one specifies this game, but let us suppose that it will end with the split of the surplus determined by the weights $\left(\pi_{1}, 1-\pi_{1}\right)$ if renegotiation is successful. It is also possible that the buyer will entertain beliefs about what would happen if he approaches the seller prior to $t^{*}$ instead of taking delivery right away.

Specifically, consider the buyer's trade option with price $p$ if she takes delivery. Fix the following extensive form after the investment has been made:

- The buyer chooses to take delivery or not. If she does, the game ends and payoffs (disregarding investment cost) are $(5-p, 3+p)$. If she does not, the game proceeds to the next stage.

- Renegotiation: let us compress the possible multi-stage bargaining game of this stage into the following 2-by-2 game in normal form, in which the buyer and seller can play "tough" (T) or "soft" (S). Payoffs are as follows: if the buyer plays $\mathrm{S},(5-p, 3+p)$ regardless of the seller's action; if she plays $\mathrm{T}$, payoffs are $\left(8 \pi_{1}, 8\left(1-\pi_{1}\right)\right)$ if the seller plays $\mathrm{S}$ and $(0,0)$ if the seller plays $\mathrm{T}$.

The interpretation of the payoffs written for the renegotiation subgame is clear. Renegotiation is not effective if the buyer plays soft. However, if she is tough and insists on not taking delivery, the seller may give in, in which case renegotiation is successful and the surplus is split using the weights $\left(\pi_{1}, 1-\pi_{1}\right)$, or he also plays tough, in which case they will disagree and trade will not take place.

Note how for a fixed $\pi_{1}$ the renegotiation subgame has multiple equilibrium payoffs if and only if $5-p<8 \pi_{1}$. This multiplicity of equilibria in the renegotiation subgame creates multiple equilibrium payoffs in the entire game that starts after the seller has made the investment. Which of the multiple equilibria will be played is entirely a matter of players' expectations. First, there is an equilibrium of the trade option contract in which the buyer takes delivery because he anticipates the (S,T) equilibrium in the renegotiation subgame. The second equilibrium, however, has the buyer not taking delivery, followed by the $(\mathrm{T}, \mathrm{S})$ equilibrium in the subgame.

In sum, taking into account the possibility of the inefficient outcome $(0,0)$ should make one consider full implementation, and the conclusion one reaches then is that the buyer's option 
contract fails to achieve full implementation if and only if $p>5-8 \pi_{1}$, i.e., the set of payoffs that $\mathrm{W}$ argues is added to the set identified by the MM approach (the interval $(1,5]$ if $\pi_{1}=1 / 2$ ).

To solve inefficiencies, W's replacement of MM's logic with the buyer's optimal decision in the contract seemed to have eliminated the essentiality of the constraint imposed by renegotiation. However, the buyer's decision problem in the trade options contract cannot be analyzed in isolation from his own beliefs. That is, he may entertain multiple beliefs as to how the seller will react if the buyer complains to him regarding the terms of trade; and this multiplicity of beliefs creates a serious problem from the point of view of full implementation when one is outside of the range of payoffs identified by MM.

\section{Concluding Remarks}

As avenues for further Research, $W$ is right in saying that one should pay attention to the technological detail of contract relationships. However, as we have argued, this should not be taken to mean that the durability of trading possibilities may interfere with the renegotiation of contracts. Even in a dynamic setting, as long as agents are capable of foreseeing ex post inefficiencies, the door to renegotiation is clearly open. We have also argued that W's nonforcing contracts do not constitute an intermediate paradigm between the two approaches to implementation, with and without renegotiation.

On the other hand, the difficulties may be serious if the renegotiation game admits multiple equilibrium payoffs, if agents are boundedly rational and cannot form rational expectations about the outcome of renegotiation, or if agents are immerse in an incomplete information environment. In any of these three cases, one should anticipate that the details of the renegotiation process may present different limitations to implementability from those studied by MM. Indeed, renegotiation processes would not necessarily restore ex post efficiency, thereby questioning the entire renegotiation program. 


\section{References}

Che, Y.-K. And D. Hausch (1999), "Cooperative Investments and the Value of Contracting," American Economic Review 89, 125-147.

Edlin, A. And S. Reichelstein (1996), "Holdups, Standard Breach Remedies, and Optimal Investment," American Economic Review 86, 478-501.

Hart, O. And J. Moore (1988), "Incomplete Contracts and Renegotiation," Econometrica 56, $755-785$.

MASkin, E. AND J. Moore (1999), "Implementation and Renegotiation," Review of Economic Studies 66, 39-56.

Segal, I. (1999), "Complexity and Renegotiation: A Foundation for Incomplete Contracts," Review of Economic Studies 66, 57-82.

Segal, I. And M. Whinston (2002), "The Mirrlees Approach to Mechanism Design with Renegotiation (with Applications to Hold-Up and Risk-Sharing)," Econometrica 70, 1-45.

Watson, J. (2002 revised 2003), "Contract, Mechanism Design and Technological Detail," Discussion Paper 2002-04, Department of Economics, University of California, San Diego. 